\title{
Tuning Light Absorption in Core/Shell Silicon Nanowire Photovoltaic Devices through Morphological Design
}

\section{Citation}

Kim, Sun-Kyung, Robert Watson Day, James F. Cahoon, Thomas Jan Kempa, Kyung-Deok Song, Hong-Gyu Park, and Charles M. Lieber. 2012. Tuning light absorption in core/shell silicon nanowire photovoltaic devices through morphological design. Nano Letters 12(9): 4971-4976.

\section{Published Version}

doi:10.1021/nl302578z

\section{Permanent link}

http://nrs.harvard.edu/urn-3:HUL.InstRepos:10397684

\section{Terms of Use}

This article was downloaded from Harvard University's DASH repository, and is made available under the terms and conditions applicable to Open Access Policy Articles, as set forth at http:// nrs.harvard.edu/urn-3:HUL.InstRepos:dash.current.terms-of-use\#OAP

\section{Share Your Story}

The Harvard community has made this article openly available.

Please share how this access benefits you. Submit a story.

\section{Accessibility}




\title{
Tuning Light Absorption in Core/Shell Silicon
}

\section{Nanowire Photovoltaic Devices through Morphological}

\section{Design}

Sun-Kyung Kim, ${ }^{\dagger, \perp,}$ Robert W. Day, ${ }^{\dagger,}$ James F. Cahoon, ${ }^{\dagger, ~ /, \S ~ T h o m a s ~ J . ~ K e m p a, ~}{ }^{\dagger}$

Kyung-Deok Song, ${ }^{\perp}$ Hong-Gyu Park, ${ }^{*}{ }^{\perp}$ and Charles M. Lieber ${ }^{*}+*$

${ }^{\dagger}$ Department of Chemistry and Chemical Biology and School of Engineering and Applied Sciences, Harvard University, Cambridge, Massachusetts 02138, USA, ${ }^{\perp}$ Department of Physics, Korea

University, Seoul 136-701, Republic of Korea

$\S$ These authors contributed equally to this work.

\begin{abstract}
Subwavelength diameter semiconductor nanowires can support optical resonances with anomalously large absorption cross sections, and thus tailoring these resonances to specific frequencies could enable a number of nanophotonic applications. Here, we report the design and synthesis of core/shell p-type/intrinsic/n-type (p/i/n) Si nanowires (NWs) with different sizes and cross-sectional morphologies, as well as measurement and simulation of photocurrent spectra from single-NW devices fabricated from these NW building blocks. Approximately hexagonal cross-section p/i/n coaxial NWs of various diameters $(170-380 \mathrm{~nm})$ were controllably synthesized by changing the Au catalyst diameter, which determines core diameter, as well as shell deposition time, which determines shell thickness. Measured polarization-resolved photocurrent spectra exhibit well-defined diameter dependent peaks. The corresponding external quantum efficiency (EQE) spectra calculated from these data show good quantitative agreement with finite-difference time-domain (FDTD) simulations, and
\end{abstract}


allow assignment of the observed peaks to Fabry-Perot, whispering-gallery, and complex high-order resonant absorption modes. This comparison revealed a systematic red-shift of equivalent modes as a function of increasing NW diameter, and a progressive increase in the number of resonances. In addition, tuning shell synthetic conditions to enable enhanced growth on select facets yielded NWs with approximately rectangular cross sections; analysis of transmission electron microscopy and scanning electron microscopy images demonstrate that growth of the n-type shell at $860{ }^{\circ} \mathrm{C}$ in the presence of phosphine leads to enhanced relative Si growth rates on the four $\{113\}$ facets. Notably, polarization-resolved photocurrent spectra demonstrate that at longer wavelengths the rectangular cross-section NWs have narrow and significantly larger amplitude peaks with respect to similar size hexagonal NWs. A rectangular NW with a diameter of $260 \mathrm{~nm}$ yields a dominant mode centered at 570 $\mathrm{nm}$ with near unity EQE in the transverse-electric polarized spectrum. Quantitative comparisons with FDTD simulations demonstrate that these new peaks arise from cavity modes with high-symmetry that conform to the cross-sectional morphology of the rectangular NW, resulting in low optical loss of the mode. The ability to modulate absorption with changes in nanoscale morphology by controlled synthesis represents a promising route for developing new photovoltaic and optoelectronic devices.

Keywords: facet-selective growth / solar energy / nanoelectronic device / coaxial p/i/n nanostructure / FDTD simulations / optical resonances

Strong light confinement in nanowire (NW) structures has enabled advances in diverse photonic applications from nanolasers to photovoltaic devices. ${ }^{1-16}$ For example, the optical properties of arrays of nano- and microscale wires have been exploited for enhanced light absorption in photovoltaic devices, ${ }^{16-19}$ however, the ability to substantially manipulate absorption in individual 
nanoscale structures has not been well established. In order to quantify optical resonances supported in individual NWs, scattering ${ }^{20-22}$ and absorption cross-sections ${ }^{5,6,11,23-26}$ have been measured or calculated. Significantly, measurement of the absolute external quantum efficiency (EQE) from NW devices has been reported only in limited instances. ${ }^{6,11,23}$ We recently reported an absolute EQE value of up to $\sim 1.2$ using core/shell Si NWs with a size of $\sim 300 \mathrm{~nm}^{6}$ By comparison, EQE values of $\sim 0.15$ have been reported for microscale devices based on Al-Si Schottky junctions ${ }^{23}$ and values of $\sim 1.1$ for devices with coaxial p-n junctions that included a back-side reflector. ${ }^{11}$ Several reports of relative EQE values have been reported for $\mathrm{Si}$ and Ge nanowire devices acting as photodetectors. ${ }^{5,24,25}$

Our integrated approach to understanding the role of morphology on light absorption in individual p/i/n Si NWs is illustrated in Figure 1. First, we synthesized core/shell p/i/n Si NWs of various sizes and cross-sectional morphologies through tuning of chemical vapor deposition (CVD) growth parameters, as illustrated in Figure 1A. Single NW devices were fabricated from these building blocks to probe how size and morphology influence the absorption of Si NWs. The Si NWs explored here had diameters ranging from 100 to $400 \mathrm{~nm}$ and cross-sectional morphologies varying from hexagonal to rectangular. Second, to understand the absorption features of NW cavities, we performed three-dimensional finite-difference-time-domain (3-D FDTD) simulations, wherein a normally incident planewave interacts with a Si NW cavity, as shown in Figure 1B. Lastly, we characterized the optical resonances by direct measurement of the photocurrent generated from functioning NW photovoltaic devices illuminated at discrete wavelengths from $380-800 \mathrm{~nm}$, using the optical setup depicted in Figure 1C. ${ }^{27}$ Comparison of measured transverse-electric (TE) and transverse-magnetic (TM) polarized spectra, as depicted in Supplementary Figure S1A, to those spectra obtained from FDTD simulations, allows for the assignment of resonant modes excited within the NW cavity. Notably, the FDTD simulations used here provide quantitative agreement with experiment by accurately describing both the NW cross-sectional morphology and underlying substrate. In contrast, Lorentz-Mie theory is an 
analytical formula and, as such, can only describe a circular NW in a homogeneous medium and provide qualitative agreement with experiment. $^{21,24,25}$

A
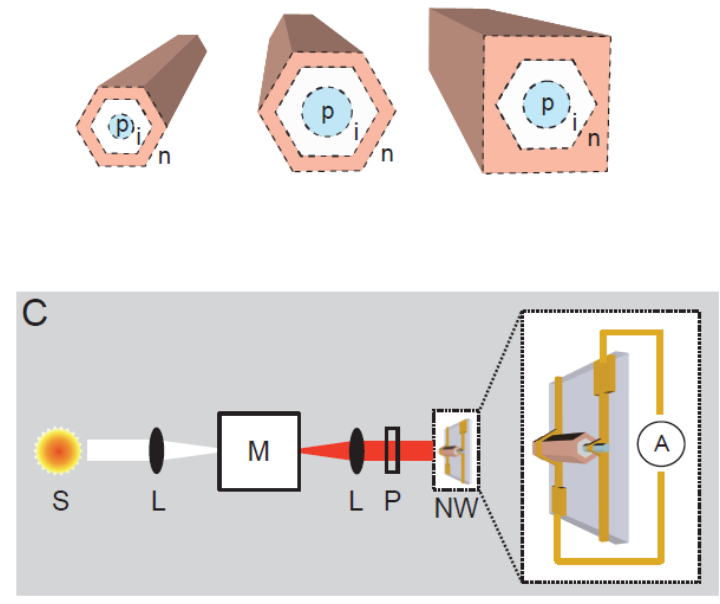

B
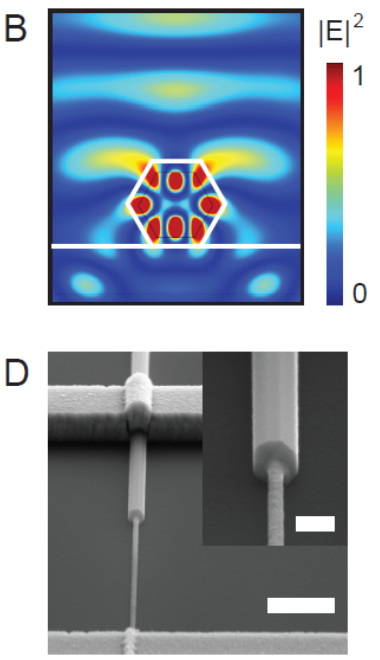

Figure 1. Overview of the experimental design and concepts. (A) Schematic illustration of designed $\mathrm{p} / \mathrm{i} / \mathrm{n} \mathrm{Si}$ NW structures, which include variations in diameter and cross-sectional morphology (hexagonal and rectangular). (B) Electric field intensity illustration of coupling between a vertically incident plane wave and highly-confined resonant modes within a Si NW. (C) Diagram of the optical setup measuring the photocurrent as a function of wavelength for single-NW photovoltaic devices. The output of Xe lamp light source $(\mathrm{S})$ passes through lenses $(\mathrm{L})$, a monochromator $(\mathrm{M})$, a polarizer (P), and then uniformly illuminates the NW device. (D) SEM image of a representative $\mathrm{p} / \mathrm{i} / \mathrm{n}$ photovoltaic device, showing metal contacts to the p-type core (bottom of image) and n-type shell (upper left of image). Scale bar, $1 \mu \mathrm{m}$. Inset, magnification of the core-shell interface showing a core diameter of $\sim 85 \mathrm{~nm}$ and shell diameter (facet to facet) of $\sim 240 \mathrm{~nm}$. Scale bar, $200 \mathrm{~nm}$.

Core/shell Si NWs were synthesized by chemical vapor deposition (CVD) using vapor-liquidsolid (VLS) growth for the p-type core ${ }^{28}$ and vapor-solid (VS) growth for intrinsic and n-type shells. ${ }^{6}$ 
In general, we have synthesized $\mathrm{p} / \mathrm{i} / \mathrm{n}$ structures of different sizes by varying the diameter of the $\mathrm{p}$-type core and thickness of the intrinsic shell. ${ }^{29}$ Control over rectangular morphology through synthesis has not been reported previously and the proposed mechanism will be discussed below. NW photovoltaic devices were fabricated by defining metal contacts selectively to the p-type core and n-type shell following selective etching of $n$ - and i-shells on one end of the NW, as shown in Figure 1D.

We first investigated the behavior of hexagonal cross-section $\mathrm{p} / \mathrm{i} / \mathrm{n} \mathrm{NWs}$ as a function of nominal diameters. SEM images show this approximately hexagonal cross-sectional morphology (Supplementary Figure S1B). ${ }^{6}$ Photocurrent spectra were acquired by measuring the current as the wavelength of incident light was scanned from 380 to $800 \mathrm{~nm} .^{27}$ EQE spectra were determined by dividing the photocurrent at a given wavelength by the incident photon flux at the same wavelength. The EQE spectra for devices with diameters of 170, 280, and $380 \mathrm{~nm}$ (solid black, Figure 2A), highlight several key features. First, the number of measured peaks increases with increasing NW size: 5 for the smallest device, 6 for the intermediate device, and 7 for the largest device. Second, the cut-off wavelength, the longest wavelength of a peak in the spectrum, for resonant modes shifts to longer wavelengths with increasing NW size: $565 \mathrm{~nm}$ for the smallest device, $635 \mathrm{~nm}$ for the intermediate device, and $670 \mathrm{~nm}$ for the largest device. Consequently, the increase in NW size produces a gradual increase in short-circuit current density $\left(J_{\mathrm{SC}}\right)$, and this trend is reflected in the red points plotted in Figure $2 \mathrm{~B}$ as a function of the diameter of the $\mathrm{NW} \cdot{ }^{27}$ 


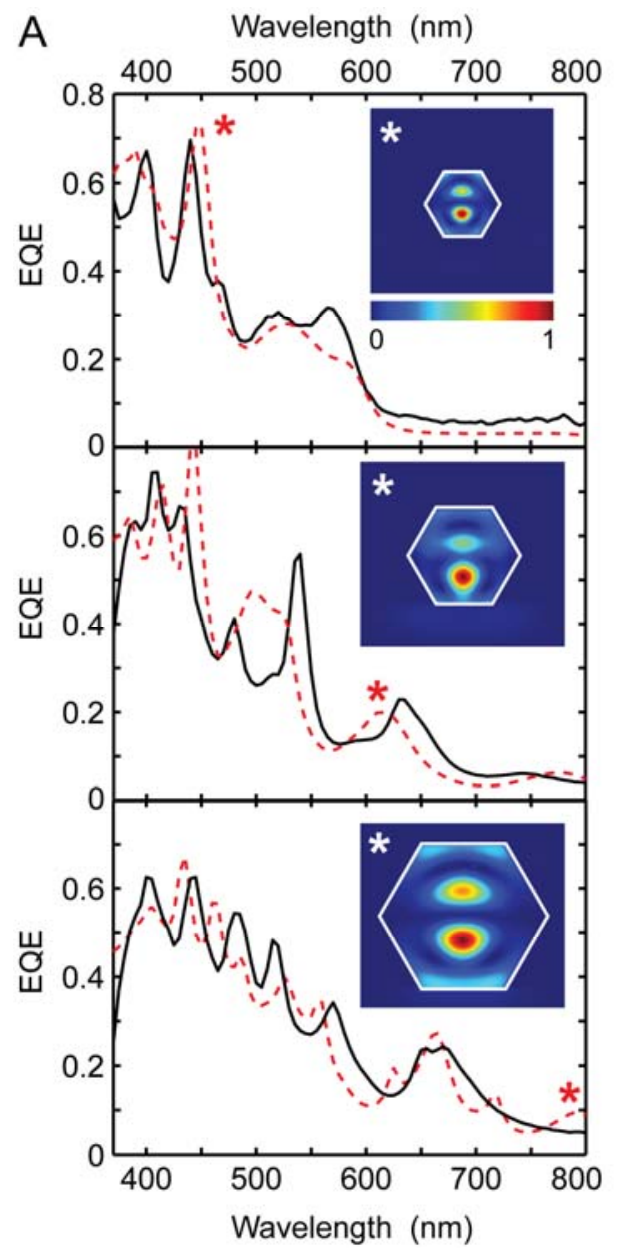

B

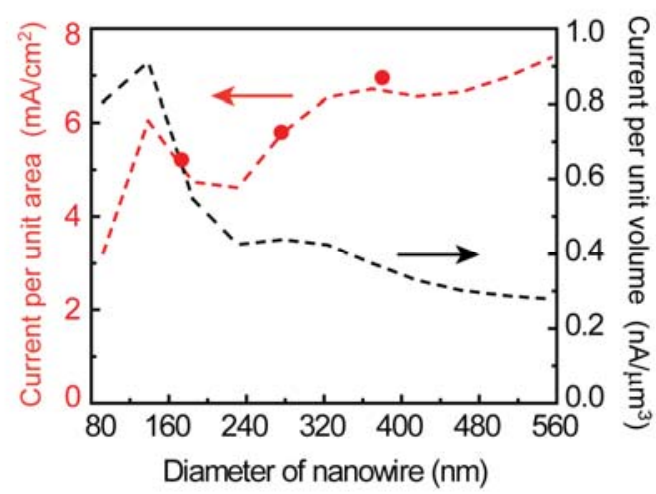

Figure 2. Size-dependent properties of hexagonal p/i/n Si NWs. (A) Experimental (solid black) and simulated (dashed red) EQE spectra for NWs with diameters of 170 (top), 280 (middle), and $380 \mathrm{~nm}$ (bottom). Insets, normalized Fabry-Perot absorption mode profiles calculated by 3-D FDTD simulations for a hexagonal NW of the corresponding diameter. These modes correspond to the peaks marked by *. (B) Size-dependent photocurrent values for NWs with diameter varying from 80 to 560 $\mathrm{nm}$, plotted per unit area (red, left-hand axis) and per unit volume (black, right-hand axis). Values are calculated from EQE data assuming the 1-sun, AM1.5G reference power spectrum. Experimental data points are shown as solid red points and simulated data as dashed lines.

Notably, the measured EQE spectra agree well with FDTD simulations (dashed red, Figure $2 \mathrm{~A}) .{ }^{30}$ Both amplitudes and wavelengths of the measured peaks were reproduced accurately by the 
simulations, thus demonstrating that the photocurrent of the working NW device is determined by the absorption characteristics of the Si NW. Additionally, the simulations show that the same absorption modes shift to longer wavelengths with increasing NW size. For instance, the peaks at 445, 620, and $795 \mathrm{~nm}$ for the small, intermediate and large sized devices, respectively, correspond to the same FabryPerot type modes with two anti-nodes in the absorption mode profile, which are marked by * and depicted in the upper, middle, and bottom insets of Figure $2 \mathrm{~A}$. The $\sim 200 \mathrm{~nm}$ increase in size produces a shift of $\sim 350 \mathrm{~nm}$ in wavelength for this specific mode.

In addition, Figure $2 \mathrm{~B}$ shows the calculated total photocurrent per unit area $\left(J_{\mathrm{SC}}\right.$, dashed red) and per unit volume (dashed black) as a function of the diameter of the NW determined from FDTD simulations. $J_{\mathrm{SC}}$ increases gradually with increasing diameter of the $\mathrm{NW}$ with the exception of a local maximum at $140 \mathrm{~nm}$ in diameter. The $J_{\mathrm{SC}}$ calculated from simulation is in excellent agreement with the $J_{\mathrm{SC}}$ from experiment (red points, Figure $2 \mathrm{~B}$ ). The increase in $J_{\mathrm{SC}}$ for larger NWs results from an increased number of absorption peaks together with resonant modes excited at long wavelengths. Enhanced optical antenna effects from smaller NW cavities account for a local maximum in $J_{\mathrm{SC}}$ at the Si diameter of $140 \mathrm{~nm}$ (dashed red, Figure 2B). ${ }^{25}$ The photocurrent per unit volume increases dramatically for devices smaller than $200 \mathrm{~nm}$ in diameter due to increasingly larger ratio of absorption cross section to physical cross section (dashed black, Figure 2B). These results highlight the unique capacity of NW structures to efficiently localize light in nanoscale volumes and to potentially enable low-cost photovoltaic devices through reduced material per device element.

To understand in more detail the enhanced absorption in these structures, we measured polarization-resolved photocurrent spectra for the devices of Figure 2A with diameters of 170, 280, and $380 \mathrm{~nm}$ (solid black, Figures 3A - 3C). The measurement shows that the spectral density and wavelength of photocurrent peaks are nearly the same in both TE and TM spectra for all NW devices, although TE EQE peaks have higher amplitudes than corresponding TM EQE peaks centered at similar wavelengths. We matched EQE peaks in the TE and TM spectra to resonant absorption mode profiles 
calculated by FDTD simulations. The corresponding absorption mode profiles reveal important features. First, different types of resonant modes are excited in the NW cavities: Fabry-Perot, whispering-gallery, and complex, high-order two-dimensional (2-D) resonant modes. Second, different types of mode profiles emerge as the size of the NW increases. For instance, in the smallest NW with a diameter of $170 \mathrm{~nm}$, only one-dimensional (1-D) Fabry-Perot modes with one or two anti-nodes appear in the spectrum (Figure 3A). As the size of a NW increases, complex 2-D modes appear in the spectrum and the number of anti-nodes in the mode profiles increases, resulting in less localized absorption distribution (Figure 3B). As NW size increases further, all types of modes including whispering-gallery modes are observed in the spectra (Figure 3C). As a result, the absorption distribution may be spatially controlled to enhance or reduce absorption in distinct regions of the NW through synthetic control of the NW size. 


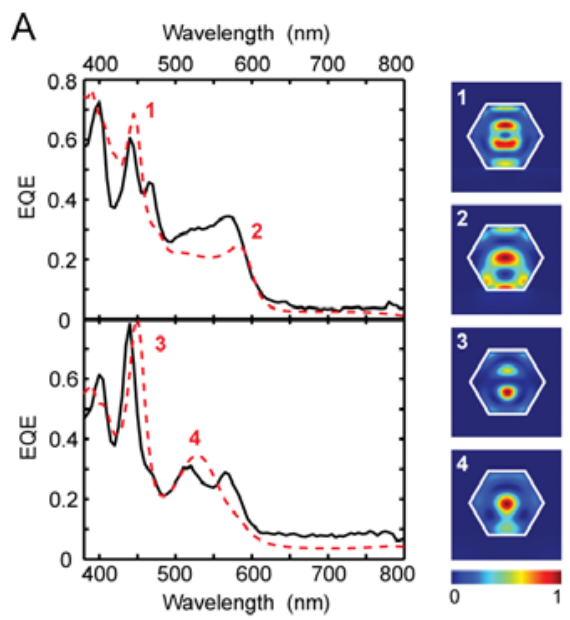

B Wavelength $(\mathrm{nm})$
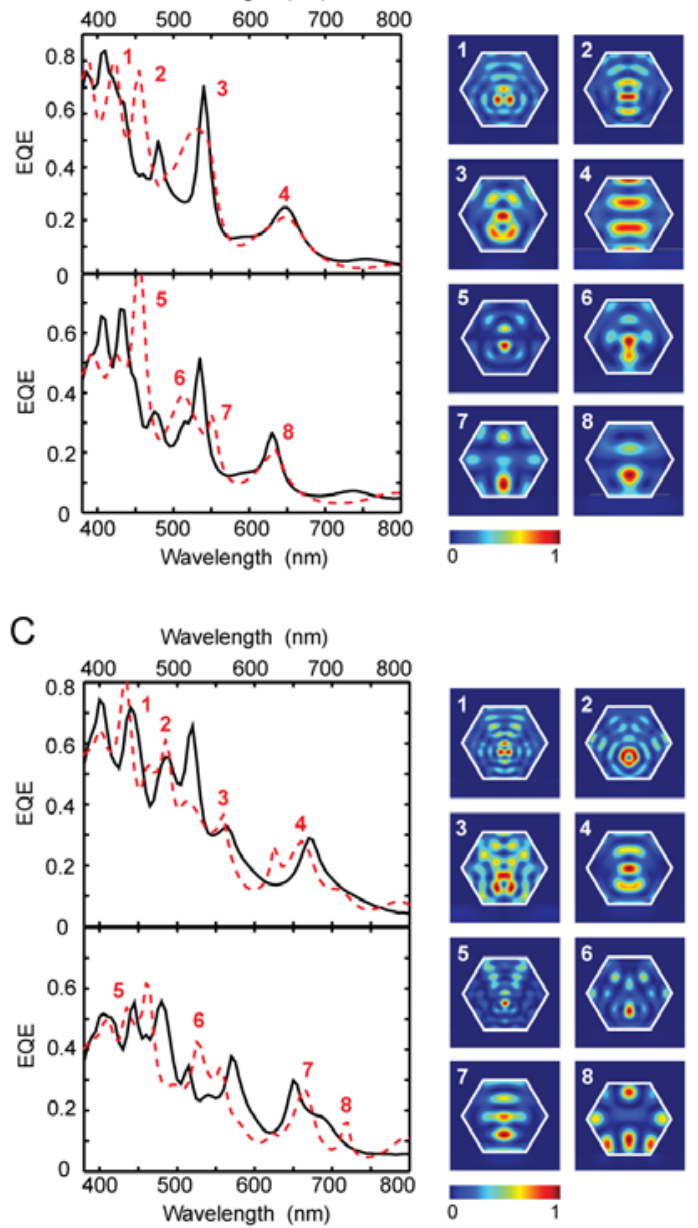

Figure 3. Polarization-resolved EQE spectra of hexagonal p/i/n Si NWs. (A), (B), (C) Left, experimental (solid black) and simulated (dashed red) EQE spectra collected with TE (top) and TM (bottom) polarization for a NW with diameter of $170 \mathrm{~nm}(\mathrm{~A}), 280 \mathrm{~nm}(\mathrm{~B})$, and $380 \mathrm{~nm}(\mathrm{C})$. Right, normalized absorption mode profiles corresponding to the peaks labeled on left. 
To characterize the effect of NW morphology on the photocurrent spectrum, we synthesized core/shell $\mathrm{p} / \mathrm{i} / \mathrm{n}$ NWs with a rectangular cross section by tuning the shell synthetic conditions to enable preferential growth on select facets. Specifically, shells were grown at $860{ }^{\circ} \mathrm{C}$ in the presence of phosphine for 40 minutes compared with $775{ }^{\circ} \mathrm{C}$ during synthesis of hexagonal cross-section NWs. A representative TEM image, as shown in Figure 4A, illustrates the approximately rectangular crosssectional morphology in contrast to the hexagonal morphology (Supplementary Figure S1B). SEM images of NWs with n-type shells grown at different growth time intervals suggest that the hexagonal cross section evolves into the rectangular cross section after extended growth of the n-type shell (Supplementary Figure S2). ${ }^{29}$ NWs with shells grown at $860{ }^{\circ} \mathrm{C}$ without phosphine retain the hexagonal morphology indicating that the higher temperature does not solely account for the rectangular morphology. Thus, we assign the core growth direction of the rectangular NWs to the same $<211>$ direction of the hexagonal NWs and hypothesize that this morphology transformation results from an increase in the relative growth rate of $\{113\}$ surfaces over the $\{111\}$ and $\{110\}$ surfaces due to presence of phosphine or some phosphorous species derived from phosphine. Phosphine has been shown to significantly alter the epitaxial growth of silicon in various ways, including a retardation of growth rate, ${ }^{31,32}$ and we suggest that a similar process occurs here. Non-equivalent growth rates of different crystal surfaces have been exploited for morphology control of, for example, CdTe nanocrystals ${ }^{33}$ and Au nanorods. ${ }^{34}$ To the best of our knowledge, our results are the first to demonstrate that similar synthetic principles can be exploited for morphology control of core/shell NWs grown by CVD. 
A
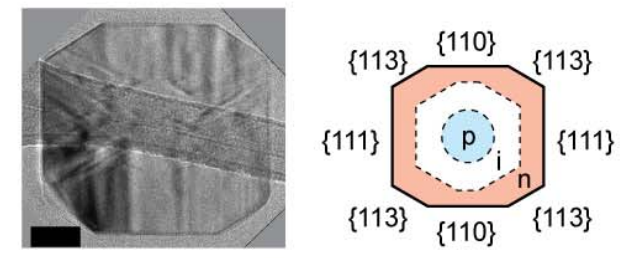

B
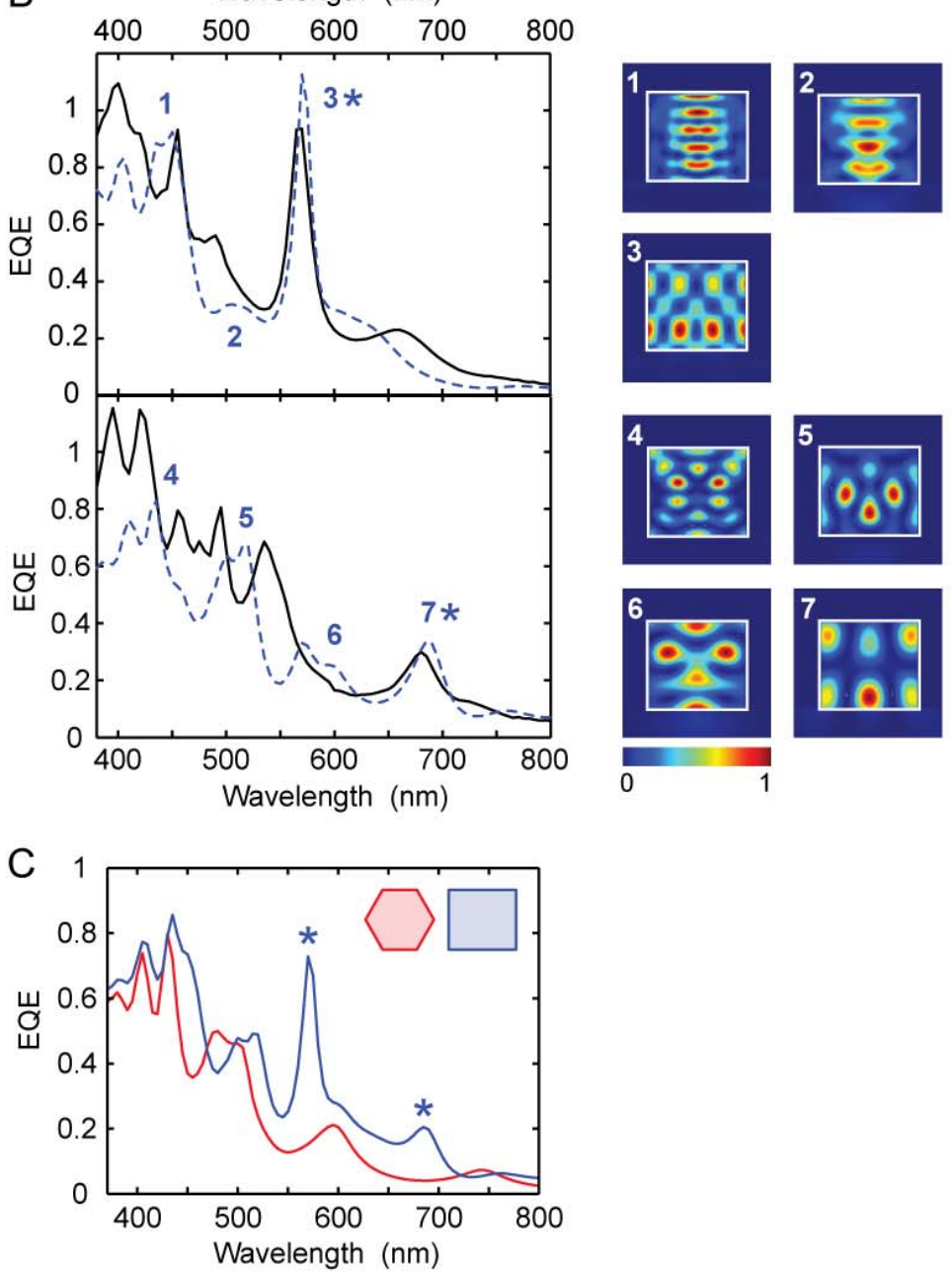

Figure 4. Fabrication and characterization of $\mathrm{p} / \mathrm{i} / \mathrm{n} \mathrm{Si} \mathrm{NWs}$ with rectangular cross-sectional morphology. (A) Left, TEM image of a rectangular p/i/n NW cross section. Scale bar, $50 \mathrm{~nm}$. Right, schematic illustration of the diode structure within the rectangular NW and with faceted surfaces assigned to $\{110\},\{113\}$, and $\{111\}$ crystal planes. (B) Left, experimental (solid black) and simulated (dashed blue) EQE spectra measured with TE (top) and TM (bottom) polarization for a rectangular NW with a diameter of $260 \mathrm{~nm}$. Absorption modes with high-order symmetry are marked by *. Right, 
normalized absorption mode profiles for the peaks labeled on left. (C) Simulated EQE spectra for a hexagonal (solid red) and rectangular (solid blue) NW with the same diameter of $260 \mathrm{~nm}$. An aspect ratio of 0.87 is used for both morphologies. Inset, schematic illustration of the two simulated structures.

Single NW photovoltaic devices were fabricated from these rectangular cross section NWs and photocurrent spectra were acquired in the same way as for hexagonal cross section NWs. The polarization-resolved EQE spectra for a rectangular NW with a diameter of $260 \mathrm{~nm}$ reveal several key features (solid black, Figure 4B). In the TE spectrum (top, Figure 4B), the peak centered at $570 \mathrm{~nm}$ shows a near unity EQE amplitude. The peak at $680 \mathrm{~nm}$ in the TM absorption spectrum (bottom, Figure 4B) shows an EQE value of $\sim 0.3$, which is significant given that crystalline Si has a very low extinction coefficient at this long wavelength. For the peak centered at $570 \mathrm{~nm}(680 \mathrm{~nm})$, an equivalent EQE value in bulk Si could only be achieved by more than $\sim 3.3 \mu \mathrm{m}(\sim 1.3 \mu \mathrm{m})$ of material. The same two high amplitude modes were also identified in the spectra of a $\sim 50 \mathrm{~nm}$ smaller rectangular NW with the same aspect ratio (Supplementary Figure S3A). These pronounced peaks were reproduced in the simulated EQE spectra (dashed blue, Figure 4B) of the rectangular NW. Notably, the pronounced peaks centered at long wavelengths correspond to high-symmetry cavity modes (marked by * and depicted in Figure 4B).

The distinct absorption properties of the rectangular NW are most apparent by comparing the simulated EQE spectrum of a rectangular NW to the spectrum of a hexagonal NW with equivalent diameter $(260 \mathrm{~nm})$ and aspect ratio (0.87), as shown in Figure 4C. The high amplitude peaks at $565 \mathrm{~nm}$ (TE) and $680 \mathrm{~nm}(\mathrm{TM})$ are observed in the EQE spectra of the rectangular NW (marked by * in Figure 4C), however the peaks at similar wavelengths in the hexagonal NW have much smaller amplitudes. Quantitative calculations of the cavity quality (Q) factors for the same TE modes centered at $\sim 600 \mathrm{~nm}$ in rectangular and hexagonal NW cavities give values of 33 and 13, respectively (Supplementary 
Figure S3B). The higher Q factor for the rectangular structure generates a narrow, high amplitude peak in the spectrum (inset, Supplementary Figure S3B). The high amplitude peaks in the rectangular NW cavity have highly symmetric mode profiles that conform to the cross-sectional morphology of the rectangular NW, resulting in low optical loss for the mode. ${ }^{35}$ Taken together, the rational design of cross-sectional morphology can provide a feasible method to enhance absorption efficiency at specific wavelengths.

In summary, we synthesized core/shell p/i/n Si NW structures with different sizes and crosssectional morphologies and demonstrated that these morphological differences affect the EQE spectra of single NW photovoltaic devices. EQE spectra showed that the number and wavelength of resonant modes increase with the size of the hexagonal NW. Comparison of experimental polarization-resolved spectra to spectra from FDTD simulations allowed for the assignment of resonant modes into three distinct types: Fabry-Perot, whispering gallery, and complex 2-D modes. In addition, we have demonstrated that enhanced facet shell growth can be exploited to evolve NW cross-sectional morphology from hexagonal to rectangular. Notably, we demonstrated with both experimental measurements and simulations that the rectangular cross-section NWs have enhanced EQE values at long wavelengths due to resonant modes excited within these high symmetry structures. Although this report focuses solely on $\mathrm{Si}$ NWs, our concepts are general and other high refractive index NW materials (e.g. Ge, GaAs, $\mathrm{PbS}$ ) can exhibit similar size and morphology-dependent optical resonances. Continued development of synthetic methods for growth of complex morphology and size-controlled NW structures of different compositions can thus lead to further advancements in the control of absorption properties in NW materials. These capabilities may have important implications for the development of ultra-thin film photovoltaic structures employing horizontal arrays of NWs and for optoelectronic devices which can utilize the tunable, wavelength and polarization-selective absorption properties of NW systems. 


\section{ASSOCIATED CONTENT}

Supporting Information. Additional information and figures. This material is available free of charge via the Internet at http://pubs.acs.org.

\section{AUTHOR INFORMATION}

Corresponding Authors.*E-mail: hgpark@korea.ac.kr and cml@cmliris.harvard.edu

Present Address. "Department of Chemistry, University of North Carolina at Chapel Hill, Chapel Hill, North Carolina 27599, USA

ACKNOWLEDGMENTS. C.M.L. acknowledges support from the Abengoa Solar New Technologies S.A. and a National Security Science and Engineering Faculty Fellow (NSSEFF) award. H.-G.P. acknowledges support of this work by Creative Research Initiatives (2012-0000242) of the National Research Foundation of Korea (NRF). J.F.C. acknowledges an Intelligence Community Postdoctoral Research Fellowship in association with Harvard University. T.J.K. and R.W.D. both acknowledge National Science Foundation Graduate Research Fellowships. 


\section{REFERENCES}

(1) Duan, X.; Huang, Y.; Agarwal, R.; Lieber, C. M. Nature 2003, 421, 241-245.

(2) Huang, M. H.; Mao, S.; Feick, H.; Yan, H.; Wu, Y.; Kind, H.; Weber, E.; Russo, R.; Yang, P. Science 2001, 292, 1897-1899.

(3) Wang, J.; Gudiksen, M. S.; Duan, X.; Cui, Y.; Lieber, C. M. Science 2001, 293, 1455-1457.

(4) Park, H.-G.; Barrelet, C. J.; Wu, Y.; Tian, B.; Qian, F.; Lieber, C. M. Nat. Photonics 2008, 2, $622-626$.

(5) Cao, L. Y.; Park, J. S.; Fan, P. Y.; Clemens, B.; Brongersma, M. L. Nano Lett. 2010, 10, 12291233.

(6) Kempa, T. J.; Cahoon, J. F.; Kim, S.-K.; Day, R. W.; Bell, D. C.; Park. H.-G.; Lieber, C. M. Proc. Natl. Acad. Sci. USA. 2012, 109, 1409-1412.

(7) Tian, B. Z.; Zheng, X.; Kempa, T. J.; Fang, Y.; Yu, N.; Yu, G.; Huang, J.; Lieber, C. M. Nature 2007, 449, 885-889.

(8) Kempa, T. J.; Tian, B.; Kim, D. R.; Hu, J.; Zheng, X.; Lieber, C. M. Nano Lett. 2008, 8, 34563460.

(9) Dong, Y.; Tian, B.; Kempa, T. J; Lieber, C. M. Nano Lett. 2009, 9, 2183-2187.

(10) Zhu, J.; Hsu, C.-M.; Yu, Z.; Fan, S.; Cui, Y. Nano Lett. 2010, 10, 1979-1984.

(11) Kelzenberg, M. D.; Turner-Evans, D. B.; Putnam, M. C.; Boettcher, S. W.; Briggs, R. M.; Baek, J. Y.; Lewis, N. S.; Atwater, H. A. Energy Environ. Sci. 2011, 4, 866-871.

(12) Fan, Z.; Razavi, H.; Do, J.; Moriwaki, A.; Ergen, O.; Chueh, Y.-L.; Leu, P. W.; Ho, J. C.;

Takahashi, T.; Reichertz, L. A.; Neale, S.; Yu, K.; Wu, M.; Ager, J. W.; Javey, A. Nat. Mater. 2009, 8, $648-653$.

(13) Tang, J.; Huo, Z.; Brittman, S.; Yang, P. Nat. Nanotech. 2011, 6, 568-572.

(14) Garnett, E.; Yang, P. Nano Lett. 2010, 10, 1082-1087. 
(15) Law, M.; Greene, L. E.; Johnson, J. C.; Saykally, R.; Yang, P. Nat. Mater. 2005, 4, 455-459.

(16) Ding, I.-K.; Zhu, J.; Cai, W.; Moon, S.-J.; Cai, N.; Wang, P.; Zakeeruddin, S. M.; Gratzel, M.; Brongersma, M. L.; Cui, Y.; McGehee, M. D. Adv. Energy Mater. 2011, 1, 52-57.

(17) Kelzenberg, M. D.; Boettcher, S. W.; Petykiewicz, J. A.; Turner-Evans, D. B.; Putnam, M. C.; Warren, E. L.; Spurgeon, J. M.; Briggs, R. M.; Lewis, N. S.; Atwater, H. A. Nat. Mater. 2010, 9, 239244.

(18) Putnam, M. C.; Boettcher, S. W.; Kelzenberg, M. D.; Turner-Evans, D. B.; Spurgeon, J. M.;

Warren, E. L.; Briggs, R. M.; Lewis, N. S.; Atwater, H. A. Energy Environ. Sci. 2010, 3, 1037-1041.

(19) Chueh, Y. L.; Fan, Z.; Takei, K.; Ko, H.; Kapadia, R.; Rathore, A. A.; Miller, N.; Yu, K.; Wu, M.; Haller, E. E.; Javey, A. Nano Lett. 2010, 10, 520-523.

(20) Cao, L. Y.; Fan, P. Y.; Brongersma, M. L. Nano Lett. 2011, 11, 1463-1468.

(21) Bronstrup, G.; Jahr, N.; Leiterer, C.; Csaki, A.; Fritzsche, W.; Christiansen, S. ACS Nano 2010, 4, $7113-7122$.

(22) Seo, K.; Wober, M.; Steinvurzel, P.; Schonbrunt, E.; Dan, Y.; Ellenbogen, T.; Crozier, K. B. Nano Lett. 2011, 11, 1851-1856.

(23) Kelzenberg, M. D., Turner-Evans, D. B.; Kayes, B. M.; Filler, M. A.; Putnam, M. C.; Lewis, N. S.; Atwater, H. A. Nano Lett. 2008, 8, 710-714.

(24) Cao, L.; White, J. S.; Park, J.-S.; Schuller, J. A., Clemens, B. M.; Brongersma, M. L.; Nat. Mater. 2009, 8, 643-647.

(25) Cao, L.; Fan, P.; Vasudev, A. P.; White, J. S.; Yu, Z.; Cai, W.; Schuller, J. A.; Fan, S.;

Brongersma, M. L. Nano Lett. 2010, 10, 439-445.

(26) Barnard, E. S.; Pala, R. A.; Brongersma, M. L. Nat. Nanotech. 2011, 6, 588-593.

(27) A solar simulator (150W, Newport Oriel) with AM 1.5G filter and calibrated 1-sun intensity was used in conjunction with a probe station (TTP-4, Desert Cryogenics) and semiconductor parameter 
analyzer (4156C, Agilent Technologies) to obtain all device transport characteristics. For EQE spectra, devices were wire-bonded to a chip carrier and interfaced with a home-built optical setup utilizing the solar simulator with AM1.5G filter as illumination source and a spectrometer (SpectraPro 300i, Acton Research) for narrowband illumination of the NW devices concurrent with measurement of $J_{\mathrm{SC}}$ at each wavelength. For comparison between experimental and simulated $J_{\mathrm{SC}}$ values, the experimental $J_{\mathrm{SC}}$ values were determined from the experimental EQE spectra from $380-800 \mathrm{~nm}$ and the reference AM1.5G spectrum. Ultra-thin NW cross-sections for TEM were prepared by embedding NWs within epoxy resin (Epo-Tek 353ND, Epoxy Technology) and then microtoming 40 nm-thick sections using a diamond blade.

(28) Schmidt, V.; Wittemann, J. V.; Senz, S.; Gosele, U. Adv. Mater. 2009, 21, 2681-2702.

(29) Silicon core/shell NWs were synthesized in a quartz tube furnace connected to a vacuum pump (base pressure $3 \times 10^{-3}$ Torr) and gas manifold with silane $\left(\mathrm{SiH}_{4}\right)$, diborane $\left(\mathrm{B}_{2} \mathrm{H}_{6} ; 100 \mathrm{ppm} \mathrm{in} \mathrm{H}_{2}\right)$, phosphine $\left(\mathrm{PH}_{3} ; 1000\right.$ ppm in $\left.\mathrm{H}_{2}\right)$, and $\mathrm{H}_{2}$ (99.999\% semiconductor grade). Crystalline Si p-type NW cores were synthesized by Au-catalyzed VLS growth using $50 \mathrm{~nm}$ Au nanoparticles (Ted Pella) for small NWs and $100 \mathrm{~nm}$ nanoparticles for intermediate and large NWs. Nanoparticles were dispersed on Si wafers with $600 \mathrm{~nm}$ thermal oxide (Nova Electronic Materials) and placed in the quartz tube furnace for core growth for 2.5 hours at $450{ }^{\circ} \mathrm{C}$ with a pressure of 40 Torr using flow rates of 1 standard cubic centimeter per minute ( $\mathrm{sccm}$ ) $\mathrm{SiH}_{4}, 10 \mathrm{sccm} \mathrm{B}_{2} \mathrm{H}_{6}$, and $60 \mathrm{sccm} \mathrm{H}_{2}$. Shell growth was performed in the same reactor using VS growth conditions at $775^{\circ} \mathrm{C}$ with a pressure of 25 Torr and $0.15 \mathrm{sccm} \mathrm{SiH}_{4}$ and $60 \mathrm{sccm} \mathrm{H}_{2}$ for intrinsic shells and additional $0.75 \mathrm{sccm} \mathrm{PH}_{3}$ for n-type shells. Typical shell growth times were 25 min for intrinsic and 15 min for n-type. Rectangular NWs were produced by increasing the time and temperature used for the n-type shell to $40 \mathrm{~min}$ and $860{ }^{\circ} \mathrm{C}$, respectively. Conformal $\mathrm{SiO}_{2}$ dielectric shells were deposited via plasma enhanced chemical vapor deposition (PECVD) over as-grown NWs on the growth substrate. NWs were dry transferred to Si substrates coated with $100 \mathrm{~nm}$ thermal oxide and $200 \mathrm{~nm} \mathrm{Si}{ }_{3} \mathrm{~N}_{4}$ (University Wafer). SU-8 was 
patterned by electron beam lithography over NWs to act as an etch mask during wet-chemical etch of NWs with buffered $\mathrm{HF}(\mathrm{BHF})$ to remove $\mathrm{SiO}_{2}$ and with $\mathrm{KOH}\left(60{ }^{\circ} \mathrm{C}\right)$ to remove the $\mathrm{Si}$ shells. SU-8 was subsequently removed with a UV/Ozone asher, and residual PECVD deposited $\mathrm{SiO}_{2}$ was removed with a BHF etch. Electron-beam lithography and thermal evaporation of $\sim 350 \mathrm{~nm}$-thick Ti/Pd was used to form selective contacts to the etched (p-type) Si core and un-etched (n-type) Si shell.

(30) For a vertically incident plane wave with TE and TM polarizations, the absorption cross section of a NW was determined from integrating $J \cdot \mathrm{E}$ at each grid point over one optical period, where $J$ and $\mathrm{E}$ are the polarization current density and electric field, respectively. The absorption efficiency was defined by the ratio of the NW absorption cross section to the projected area. EQE was calculated by multiplying the absorption efficiency by internal quantum efficiency (IQE), where IQE was assumed to be 0.8 . The short circuit current density $\left(J_{\mathrm{SC}}\right)$ at a specific wavelength was calculated as follows: $J_{\mathrm{SC}}(\lambda)$ $=\mathrm{EQE} \times\left(\right.$ spectral irradiance of AM $1.5 \mathrm{G}$ spectrum at 1 -sun solar intensity) $\times \lambda / 1.24$. The total $J_{\mathrm{SC}}$ was obtained by integrating $J_{\mathrm{SC}}(\lambda)$ over the wavelength range of $380-800 \mathrm{~nm}$. For hexagonal (rectangular) NWs, a spatial resolution of $5 / \sqrt{3}(5), 5$, and $5 \mathrm{~nm}$ for $\mathrm{x}, \mathrm{y}$ and $\mathrm{z}$, respectively, was used to represent the volume element for our hexagonal (rectangular) cross section of a NW, where y lies along the NW axis and $\mathrm{z}$ lies along the propagation direction of the incident plane wave. All NW simulations included the same substrates used in the experiment.

(31) Fissel, A.; Richter, W. Mater. Sci. Eng. B 2000, 73, 163-167.

(32) Cho, B.; Bareño, J.; Foo, Y. L.; Hong, S.; Spila, T.; Petrov, I.; Greene, J. E. J. Appl. Phys. 2008, $103,123530-1-123530-10$.

(33) Manna, L.; Milliron, D. J.; Meisel, A.; Scher, E. C; Alivisatos, A. P. Nat. Mater. 2003, 2, 382385.

(34) Murphy, C. J.; Sau, T. K.; Gole, A. M.; Orendorff, C. J.; Gao, J.; Gou, L.; Hunyadi, S. E.; Li, T. J. Phys. Chem. B 2005, 109, 13857-13870. 
(35) Ryu, H.-Y.; Kim, S.-H.; Park, H.-G.; Hwang, J.-K.; Lee, Y.-H. Appl. Phys. Lett. 2002, 80, 38833885. 
Table of Contents Graphic
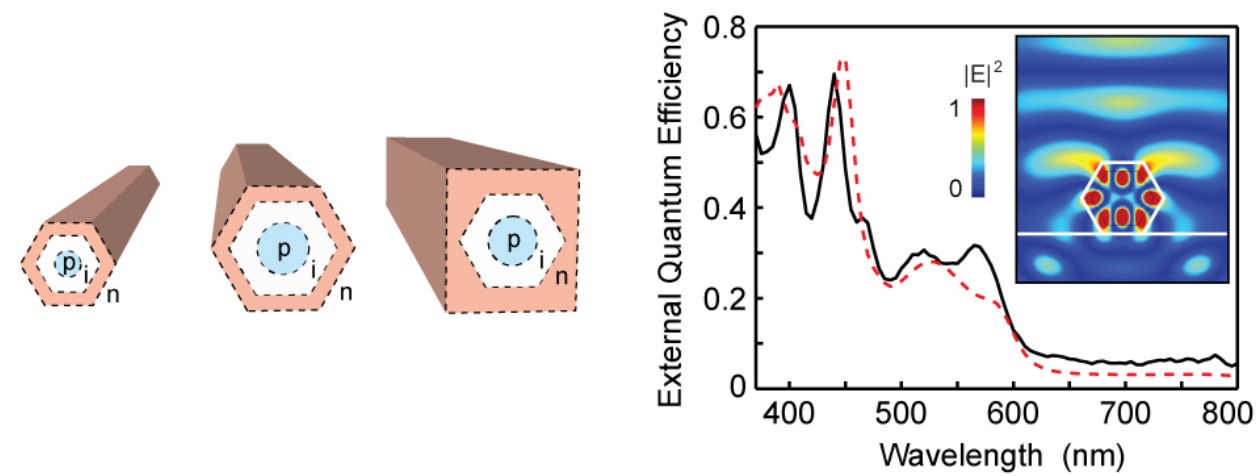
Supporting Information for:

\section{Tuning Light Absorption in Core/Shell Silicon Nanowire Photovoltaic Devices through Morphological Design}

Sun-Kyung Kim, Robert W. Day, James F. Cahoon, Thomas J. Kempa, Kyung-Deok Song,

Hong-Gyu Park, Charles M. Lieber

This file includes:

Supplementary Figures S1 - S3 


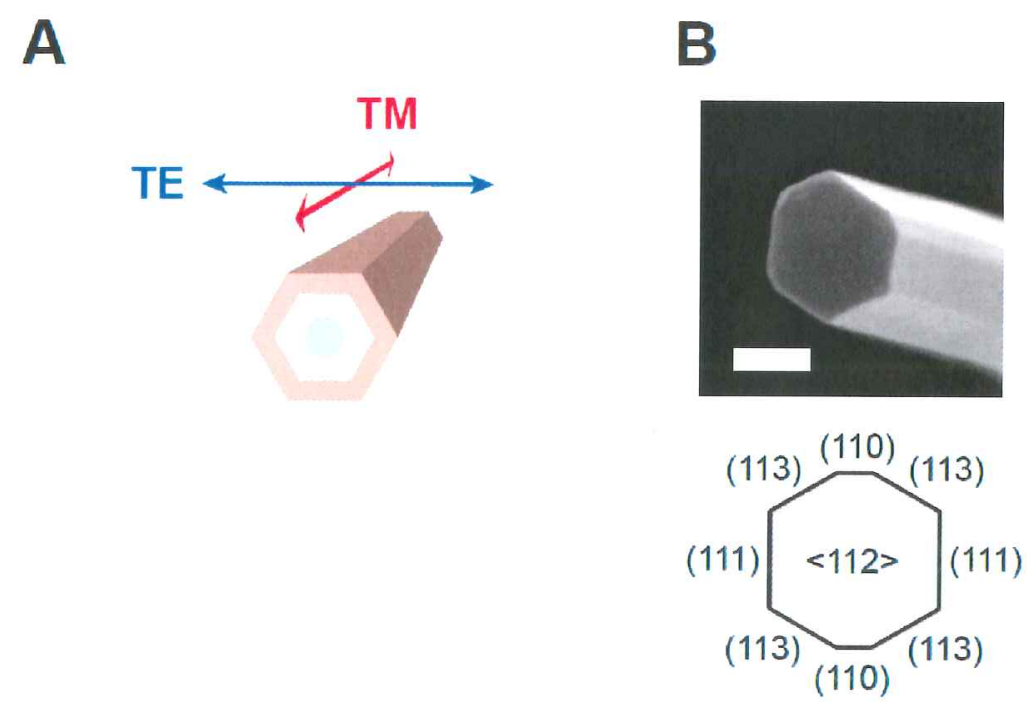

Figure S1. Characterization of hexagonal p/i/n Si NWs. (A) Schematic illustration of the electric field vector for TE and TM polarizations. (B) SEM image (top) of a p/i/n NW showing the approximately hexagonal cross-sectional morphology of the NW. Scale bar, $200 \mathrm{~nm}$. Schematic illustration (bottom) of the NW cross-sectional morphology assigning faceted surfaces to $\{110\}$, $\{113\}$, and $\{111\}$ crystal planes. 


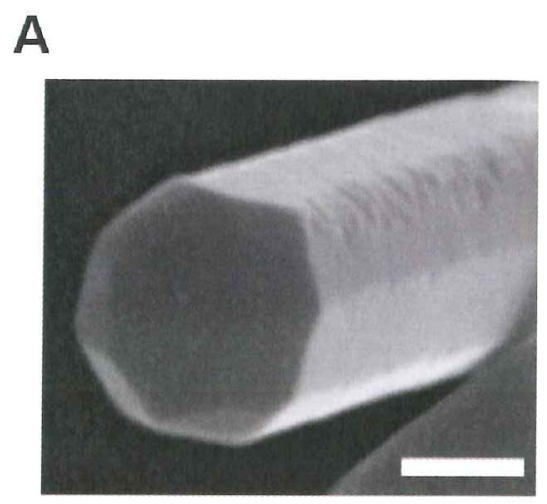

B

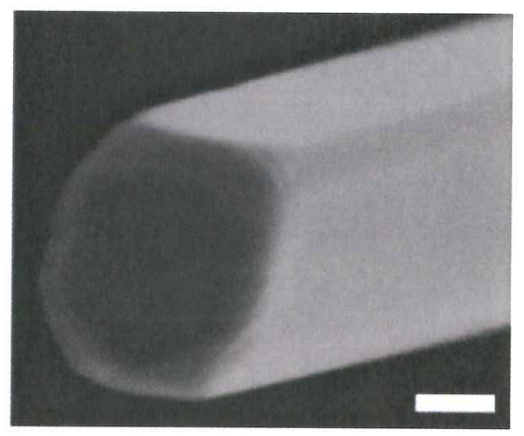

Figure S2. Characterization of p/i/n Si NWs grown with phosphine during shell growth. (A) SEM image of a pli/n NW with $n$-shell grown for 25 minutes at $860^{\circ} \mathrm{C}$. (B) SEM image of an approximately rectangular p/i/in NW with n-shell grown for 40 minutes at $860^{\circ} \mathrm{C}$. Scale bar, 100 $\mathrm{nm}$. 

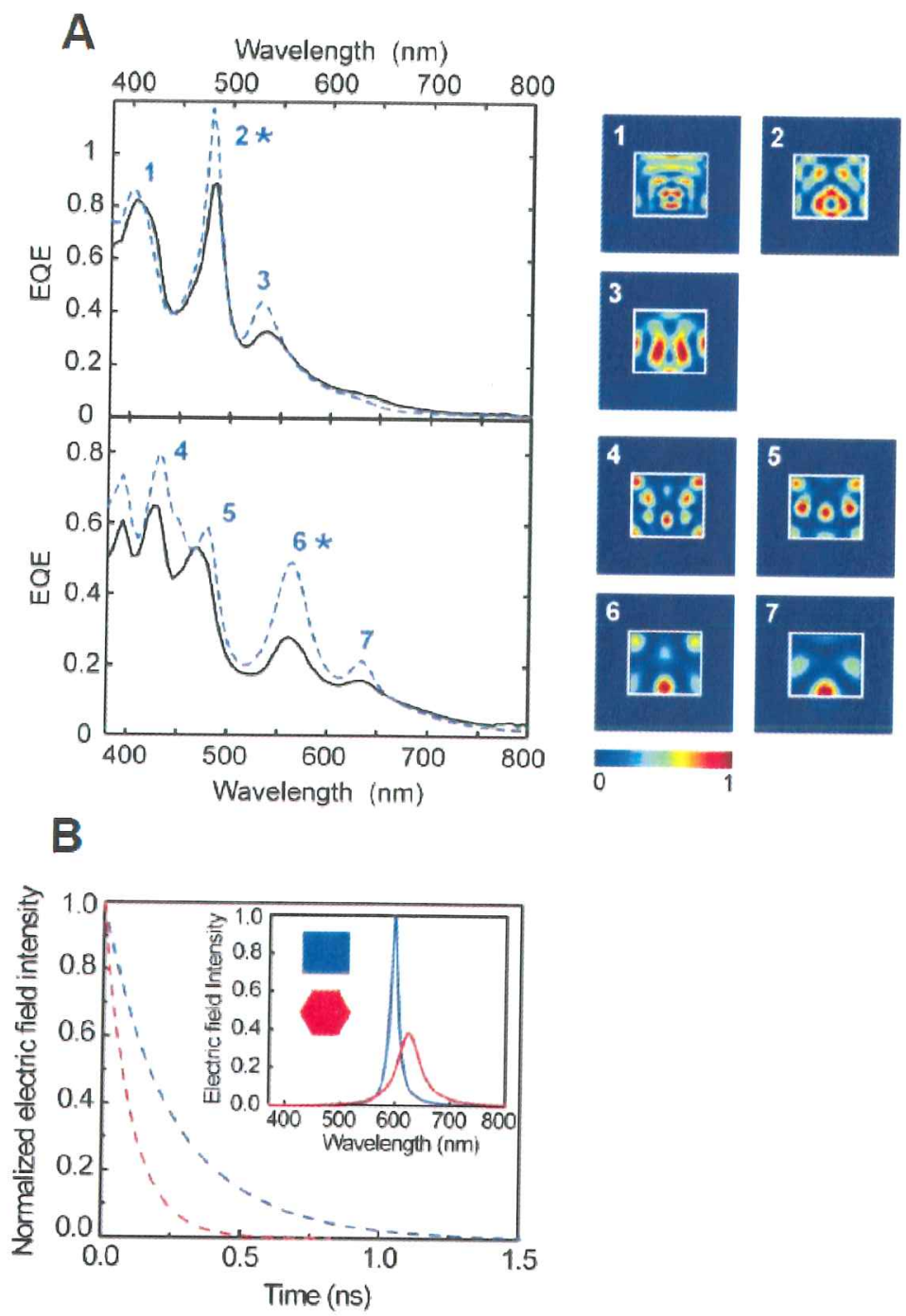

Figure S3. Polarization-resolved EQE spectra of a small rectangular NW and comparison of peak widths in hexagonal and rectangular NWs. (A) Left, experimental (solid black) and simulated (dashed blue) EQE spectra collected with TE (top) and TM (bottom) polarization for a rectangular p/i/n NW with a diameter of $\sim 200 \mathrm{~nm}$. Absorption modes with high symmetry are marked by *. Right, absorption mode profiles corresponding to the peaks labeled on left. (B) Time-dependent decay of electric field intensity for the resonant mode centered at $\sim 600 \mathrm{~nm}$ in Si NW cavities with hexagonal (dashed red) and rectangular (dashed blue) morphology and diameter of $280 \mathrm{~nm}$. Inset, Fourier transforms of the time-dependent signals, showing the narrow, high amplitude mode from the rectangular NW (solid blue) contrasting with the broadened and low one from the hexagonal NW (solid red). 